\title{
BUSCA PELA "MUSCULARIDADE" E VARIÁVEIS ASSOCIADAS EM ADULTOS JOVENS
}

\author{
DRIVE FOR MUSCULARITY AND ASSOCIATED VARIABLES IN YOUNG ADULTS
}

Artigo Original

BÚSQUEDA POR LA "MUSCULARIDAD" Y VARIABLES ASOCIADAS EN ADULTOS JÓVENES

Pedro Henrique Berbert de Carvalho' (Educador Físico)

Fernanda da Costa Oliveira ${ }^{2}$

(Educadora Física)

Clara Mockdece Neves ${ }^{3}$

(Educadora Física)

Juliana Fernandes Filgueiras Meireles (Educadora Física)

Vinícius Granato Valverdu Lopes ${ }^{4}$

(Educador Físico)

Maria Elisa Caputo Ferreira ${ }^{3}$

(Educadora Física)

1. Universidade Federal de Juiz de Fora (UFJF), Campus Governador Valadares, Governador Valadares, MG, Brasil.

2. Faculdade de Educação Física e Desportos (UFJF), Programa de Pós-Graduação em Educação Física, Juiz de Fora, MG, Brasil.

3. Instituto de Ciências Humanas (UFJF), Programa de Pós-Graduação em Psicologia, Juiz de Fora,

MG, Brasil.

4. Universidade Federal de Juiz de Fora (UFJF), Curso de Graduação em Educação Física, Juiz de Fora, MG, Brasil.

\section{Correspondência:}

Rua José Lourenço Kelmer, s/n. Campus Universitário. Bairro: São Pedro, Juiz de Fora, MG, Brasil. 36036 900. pedro.berbert@ufjf.edu.br

\section{RESUMO}

Introdução: A busca pela "muscularidade" parece estar associada a diversas variáveis que impactam a saúde e a qualidade de vida dos sujeitos. No entanto, há uma carência de estudos sobre o construto, um baixo número de instrumentos de medida específicos para sua avaliação, além de reduzida avaliação de indivíduos do sexo masculino. Objetivo: Avaliar a busca pela "muscularidade" e associá-la a sintomas depressivos, autoestima, comportamentos de risco de transtornos alimentares e ao grau de comprometimento psicológico com o exercício físico. Métodos: Realizou-se um estudo transversal com 246 jovens adultos do sexo masculino. Foram utilizados instrumentos de autorrelato para avaliar a busca pela "muscularidade" (Drive for Muscularity Scale), autoestima (Rosenberg Self-Esteem Scale), sintomas depressivos (Beck Depression Inventory), comportamentos de risco de transtornos alimentares (Eating Attitudes Test-26) e o grau de comprometimento psicológico com o exercício físico (Commitment Exercise Scale). Foi utilizado o teste de associação de Pearson para verificar relação entre as variáveis do estudo e Análise de Regressão Linear Múltipla (forward) para verificação do quanto as variáveis do estudo influenciam na busca pela "muscularidade". Resultados: A busca pela "muscularidade" esteve associada à autoestima $\left(r_{\text {pearson }}=0,13 ; p<0,05\right)$, aos comportamentos de risco de transtornos alimentares $\left(r_{\text {pearson }}=0,20 ; p<0,05\right)$ e ao grau de comprometimento psicológico ao exercício $\left(r_{\text {pearson }}=0,62 ; p<0,05\right)$. O grau de comprometimento psicológico com o exercício explicou 38,4\% da variância total da busca pela "muscularidade", 12,3\% da insatisfação com a "muscularidade" e 51,0\% dos comportamentos orientados para a "muscularidade". Conclusão: A busca pela "muscularidade" está associada à baixa autoestima, aos comportamentos de risco de transtornos alimentares e, em especial, ao grau de comprometimento psicológico com o exercício físico, o que alerta profissionais de saúde sobre os riscos da busca excessiva pelo ideal de corpo hipermusculoso e comportamentos deletérios à saúde decorrentes dessa busca.

Descritores: imagem corporal; transtornos da alimentação; autoimagem; depressão.

\section{ABSTRACT}

Introduction: Drive for muscularity seems to be associated with several variables that affect the health and quality of life of individuals. However, there is a lack of studies on the construct, a low number of specific measuring instruments, and few evaluations of males. Objectives: To evaluate the drive for muscularity and associate it with depressive symptoms, self-esteem, risk behaviors for eating disorders and the degree of psychological commitment to exercise. Methods: A cross-sectional study was conducted with 246 young male adults. Self-report instruments were used to assess the drive for muscularity (Drive for Muscularity Scale), self-esteem (Rosenberg Self-Esteem Scale), depressive symptoms (Beck Depression Inventory), risk behavior for eating disorders (Eating Attitudes Test-26), and the degree of psychological commitment to exercise (Commitment Exercise Scale). Pearson's association test was used to verify the relationship between the study variables, and Multiple Linear Regression Analysis (forward) to evaluate how much the study variables influence the drive for muscularity. Results: The drive for muscularity was associated with self-esteem ( $\left.r_{\text {pearson }}=0.13 ; p<0.05\right)$, risk behavior for eating disorders $\left(r_{\text {pearson }}=0.20 ; p<0.05\right)$ and the degree of psychological commitment to exercise $\left(r_{\text {pearson }}=\right.$ $0.62 ; p<0.05)$. The degree of psychological commitment to exercise explained $38.4 \%$ of the total variance in the drive for muscularity, $12.3 \%$ of dissatisfaction with muscularity, and $51.0 \%$ of the muscularity-oriented behaviors. Conclusion: Drive for muscularity is associated with low self-esteem, risk behaviors for eating disorders and, in particular, with the degree of psychological commitment to physical exercise, which warn health professionals about the risks of excessive pursuit of the ideal hyper muscled body and deleterious behaviors to health resulting from this pursuit.

Keywords: body image; eating disorders; self concept; depression.

\section{RESUMEN}

Introducción: La búsqueda de la "muscularidad" parece estar asociada con diversas variables que afectan a la salud y calidad de vida de los individuos. Sin embargo, faltan estudios sobre este constructo, un bajo número de instrumentos de medición específicos para su evaluación, además de la reducida evaluación de los individuos del sexo masculino. Objetivo: Evaluar la búsqueda por la "muscularidad" y asociarla con síntomas de la depresión, la autoestima, las conductas de risco para los trastornos de alimentación y el grado de compromiso psicológico para el ejercicio. Métodos: Se realizó un estudio transversal con 246 jóvenes adultos del sexo masculino. Se utilizaron instrumentos de autoinforme para evaluar la búsqueda por la "muscularidad" (Drive for Muscularity Scale), la autoestima (Rosenberg Self-Esteem Scale), los sintomas depresivos (Beck Depression Inventory), los comportamientos de riesgo para los trastornos alimentarios (Eating Attitudes Test-26) y grado de compromiso psicológico para ejercicio (Commitment Exercise Scale). 
La prueba de correlación de Pearson fue utilizada para verificar la relación entre las variables del estudio y análisis de regresión lineal múltiple (forward) para comprobar cuánto las variables del estudio influyen en la búsqueda por la "muscularidad". Resultados: La búsqueda por la "muscularidad" se asoció con la autoestima ( $\left.r_{\text {pearson }}=0,13 ; p<0,05\right)$, los comportamientos de riesgo para los trastornos de la alimentación $\left(r_{\text {pearson }}=0,20 ; p<0,05\right)$, yel grado de compromiso psicológico para el ejercicio ( $\left.r_{\text {pearson }}=0,62 ; p<0,05\right)$. El grado de compromiso psicológico para el ejercicio explicó 38,4\% de la varianza total de la búsqueda por la "muscularidad", el 12,3\% de insatisfacción con la "muscularidad" y el 51,0\% de los comportamientos orientados a la "muscularidad". Conclusión: La búsqueda por la "muscularidad" se asocia con una baja autoestima, conductas de riesgo de trastornos alimentarios y, en especial, el grado de compromiso psicológico para el ejercicio, que alerta a los profesionales de la salud acerca de los riesgos de la excesiva búsqueda por el ideal de cuerpo hipermusculoso y la conducta perjudicial para la salud como resultado de esta búsqueda.

Descriptores: imagen corporal; trastornos de la conducta alimentaria; autoimagen; depresión.

\section{INTRODUÇÃO}

A preocupação com a aparência física e com a imagem corporal é hoje uma realidade em diversos países ${ }^{1}$. A insatisfação com o próprio corpo, considerada um sentimento negativo geralmente relacionado ao sexo feminino ${ }^{2}$, pode estar presente também em indivíduos do sexo masculino ${ }^{3-5}$. Nestes, quando é o caso, a preocupação está voltada para a quantidade de músculos, em especial nos ombros, peitorais e braços ${ }^{4}$.

A busca pela "muscularidade", do original drive for muscularity", é um construto que expressa atitudes e comportamentos que refletem o grau de preocupação dos indivíduos com sua massa muscular ${ }^{6,7}$. Importante observar que, assim como a busca pela magreza (drive for thinness), comum entre as mulheres, a busca pela "muscularidade" pode trazer consequências negativas para a saúde física e psicológica do sujeito ${ }^{4}$.

O desejo por um corpo musculoso pode estar associado ao abuso de substâncias ergogênicas e esteroides anabólicos ${ }^{4,6}$, à depressão e baixa autoestima ${ }^{3}$, assim como à prática excessiva de exercício físico e ao grau de comprometimento psicológico ao mesmo 5 . Em resumo, a busca pela "muscularidade" é uma variável de interesse por estar associada a diversos comportamentos deletérios a saúde, inclusive com o transtorno alimentar ${ }^{3}$, porém ainda é pouco investigada.

Destaca-se ainda que o estudo sobre a imagem corporal e os transtornos alimentares não é frequente na população masculina ${ }^{4,5}$. Somado a isso, poucos são os instrumentos específicos para indivíduos do sexo masculino disponíveis para a avaliação desse construto ${ }^{5}$, o que dificulta a avaliação desses sujeitos. É frequente o estudo sobre a imagem corporal e os transtornos alimentares em praticantes de atividade física ${ }^{8-10} \mathrm{e}$ atletas $^{11-15}$. No entanto, em sua vasta maioria tais estudos são realizados com mulheres, e, mesmo quando incluem sujeitos do sexo masculino utilizam instrumentos de avaliação não específicos para essa população ${ }^{5}$.

Diante das limitações elencadas de estudos nacionais sobre imagem corporal e transtornos alimentares em indivíduos do sexo masculino, somado a carência de pesquisas acerca do construto drive for muscularity, a presente pesquisa teve como objetivo identificar associações entre a busca pela "muscularidade" e indicadores de depressão, autoestima, comportamentos de risco para transtornos alimentares e grau de comprometimento psicológico ao exercício físico em adultos jovens do sexo masculino.

\section{MÉTODOS}

Este estudo contou com a participação de adultos jovens (universitários) do sexo masculino, de uma Instituição de Ensino Superior (IES) da cidade de Juiz de Fora, Minas Gerais. A presente pesquisa foi aprovada pelo Comitê de Ética em Pesquisa (CEP) com Seres Humanos da Universidade Federal de Juiz de Fora, Brasil (protocolo n² 2193.253.2010; parecer $n^{\circ}$ 276/2010), e todos os voluntários concordaram participar do estudo por meio da assinatura do Termo de Consentimento Livre e Esclarecido.

A coleta ocorreu sempre ao início das aulas, na presença do professor, sem limite de tempo para o preenchimento dos questionários, que foram respondidos individualmente. Foram incluídos todos os sujeitos regularmente matriculados em qualquer período de cursos de graduação da IES, com idade superior a 18 anos, que aceitaram participar voluntariamente da pesquisa.

Para avaliar a busca pela "muscularidade" foi utilizada a Drive for Muscularity Scale (DMS) ${ }^{7,16}$. Trata-se de uma escala de autorrelato, composta por 15 itens, respondidos na forma Likert de seis pontos (um - sempre a seis - nunca), variando de 15 a 90 pontos. Quanto maior a pontuação obtida maiores são as atitudes e comportamentos de preocupação do indivíduo com sua "muscularidade"7. A escala foi traduzida e adaptada para jovens adultos brasileiros e obteve propriedades psicométricas adequadas ${ }^{17}$.

A DMS é formada por duas subescalas: insatisfação com a "muscularidade" (exemplo, "Eu gostaria de ser mais musculoso") e comportamentos orientados para a "muscularidade" (exemplos, "Levanto pesos para aumentar meus músculos" ou "Uso suplementos energéticos ou de proteína") ${ }^{17}$. A consistência interna (coeficiente alpha [a] de Cronbach) ${ }^{18}$ obtido para a DMS nesse estudo correspondeu a 0,89.

A autoestima foi avaliada pela Rosenberg Self-Esteem Scale (RSS) ${ }^{19}$. A RSS é uma escala composta por 10 itens, no formato Likert de quatro pontos (um = discordo totalmente a quatro = concordo totalmente), em que as afirmações fazem referências a um conjunto de sentimentos de autoestima e de autoaceitação. 0 escore varia de 10 a 40 pontos e, quanto maior o valor obtido maior o nível de autoestima do indivíduo. Para a pesquisa foi utilizada a versão brasileira validada por Hutz ${ }^{20}$. A RSS apresentou valor de consistência interna para autoestima igual a 0,79 na presente amostra.

Os sintomas depressivos foram avaliados por meio do Beck Depression Inventory (BDI) ${ }^{21}$. O BDI consiste em um instrumento de 21 itens, variando sua intensidade de zero a três. O escore total pode variar de zero a 63 pontos e, quanto maior a pontuação, maior é o grau de depressão do indivíduo. Foi utilizada nessa pesquisa a versão em português validada por Gorestein e Andrade 22 . Foi obtido a igual a 0,82.

Como forma de avaliar os comportamentos de risco para transtornos alimentares foi utilizado o Eating Attitudes Test-26 (EAT-26) ${ }^{23}$. Trata-se de um questionário de autorrelato composto por 26 questões em escala Likert de pontos (três = sempre; dois = muitas vezes; um = às vezes; zero = poucas vezes, quase nunca e nunca), destinado à avaliação de sintomas e características de pacientes com transtornos alimentares, apresentando boas propriedades psicométricas ${ }^{24}$. Exceção se faz ao item 25 que tem escore invertido. Escores elevados são indicadores de possíveis comportamentos relacionados aos transtornos alimentares, além da presença de padrões alimentares anormais ${ }^{23,24}$. Para a presente pesquisa, a consistência interna do EAT-26 correspondeu a 0,79.

Para determinar o grau de comprometimento psicológico ao exercício físico foi utilizada a versão brasileira da Commitment Exercise Scale (CES) ${ }^{25}$. Trata-se de uma escala analógica visual composta por oito questões que remetem ao comprometimento, comportamento e a atitude do sujeito em relação ao exercício físico. Cada pergunta da 
escala possui uma linha de 155 mm com duas opções de resposta, sendo uma no início e outra no final da linha. A distância entre o começo da linha e o ponto marcado pelo respondente consiste na pontuação de cada item. O escore varia de zero a 1.240 pontos, e quanto maior a pontuação maior o grau de comprometimento psicológico do sujeito ao exercício físico ${ }^{25}$. O a obtido foi de 0,84.

Para a avaliação do estado nutricional dos participantes foi utilizado o cálculo do Índice de Massa Corporal (IMC), através dos dados de massa corporal e estatura autorreferidos pelos participantes. Os indivíduos foram agrupados de acordo com a classificação estabelecida pela Organização Mundial da Saúde ${ }^{26}$ - IMC <18,5kg/m² (baixo peso); IMC 18,5-24,9kg/m² (eutrofia); IMC 25-29,9kg/m² (sobrepeso); e IMC >29,9kg/m² (obesidade).

Para a análise dos dados foi realizada, inicialmente, a análise descritiva de todas as variáveis por meio da média, desvios-padrão, mediana, valores mínimos e máximos. Em seguida foi utilizado o teste de normalidade Komolgorov-Smirnov, não sendo identificada violação da hipótese nula. Portanto, utilizou-se o teste de associação de Pearson para verificar associação entre as variáveis do estudo. Por fim, foi realizada a Análise de Regressão Linear Múltipla Forward para verificação do quanto as variáveis do estudo influenciam na busca pela "muscularidade". Neste caso, as regressões foram realizadas também para as subescala insatisfação com a "muscularidade"e comportamentos orientados para a "muscularidade" da DMS.

Foi calculada a consistência interna, por meio do coeficiente alpha de Cronbach, de todos os instrumentos utilizados, e considerado adequado valor superior a $0,70^{18}$.

\section{RESULTADOS}

Participaram da pesquisa 246 jovens universitários com idade média de 21,29 2,71 anos, variando entre 18 e 30 anos de idade, graduandos de diversos cursos (Ciências da Computação, Direito, Engenharia Civil, Engenharia Elétrica, Filosofia, Fisioterapia, Matemática, Medicina e Psicologia). A avaliação do estado nutricional, pelo IMC, revelou uma média de 23,63 $\pm 3,49 \mathrm{~kg} / \mathrm{m}^{2}$, sendo que 5,28\% ( $\mathrm{n}=13$ ) dos estudantes encontram-se com baixo peso, 58,13\% ( $n=143)$ eutrofia, 32,52\% $(n=80)$ sobrepeso e 4,06\% ( $n=10$ ) obesidade. Os dados descritivos das variáveis analisadas podem ser verificados na Tabela 1 .

A análise de associação das variáveis pode ser observada na Tabela 2. Verifica-se associação da busca pela "muscularidade" (DMStotal) com praticamente todas as variáveis analisadas, exceto com os sintomas depressivos. A subescala insatisfação com a "muscularidade" $\left(\mathrm{DMS}_{\text {insatisfação }}\right)$ obteve associação com quase todas as variáveis, exceto com os comportamentos de risco para transtornos alimentares. Por fim, pode ser verificado na Tabela 2, associação da subescala

Tabela 1. Análise descritiva das variáveis de estudo.

\begin{tabular}{c|c|c|c}
\hline Variáveis & Média \pm DP & Mediana & Mín- Máx \\
\hline DMS $_{\text {total }}$ & $42,20 \pm 15,88$ & 40,00 & $15,00-84,00$ \\
\hline DMS $_{\text {insatisfacão }}$ & $24,24 \pm 9,36$ & 24,00 & $7,00-42,00$ \\
\hline DMS $_{\text {compartamento }}$ & $17,96 \pm 9,14$ & 15,00 & $8,00-45,00$ \\
\hline RSS & $25,04 \pm 3,71$ & 25,00 & $10,00-35,00$ \\
\hline BDI & $6,66 \pm 5,46$ & 6,00 & $0,00-32,00$ \\
\hline EAT-26 & $10,89 \pm 7,87$ & 9,00 & $0,00-51,00$ \\
\hline CES & $53,44 \pm 28,58$ & 53,55 & $0,00-145,90$ \\
\hline
\end{tabular}

DP: Desvio-padrão; DMS: Drive for Muscularity Scale; RSS: Rosenberg Self-Esteem Scale; BDI: Beck Depression Inventory EAT-26: Eating Attitudes Test-26; CES: Commitment Exercise Scale.

Tabela 2. Matriz de correlação entre os instrumentos de medida (variáveis) do estudo.

\begin{tabular}{c|c|c|c|c|c|c|c}
\hline Variáveis & $\mathbf{1}$ & $\mathbf{2}$ & $\mathbf{3}$ & $\mathbf{4}$ & $\mathbf{5}$ & $\mathbf{6}$ & $\mathbf{7}$ \\
\hline DMS $_{\text {total }}$ & 1,00 & & & & & & \\
\hline DMS $_{\text {insatisfacăo }}$ & $0,86^{*}$ & 1,00 & & & & & \\
\hline DMS $_{\text {comportamento }}$ & $0,85^{*}$ & $0,47^{*}$ & 1,00 & & & & \\
\hline RSS & $0,13^{*}$ & $0,15^{*}$ & 0,08 & 1,00 & & & \\
\hline BDI & 0,05 & $0,15^{*}$ & $-0,07$ & $0,24^{*}$ & 1,00 & & \\
\hline EAT-26 & $0,20^{*}$ & 0,12 & $0,23^{*}$ & $0,18^{*}$ & $0,15^{*}$ & 1,00 & \\
\hline CES & $0,62^{*}$ & $0,36^{*}$ & $0,72^{*}$ & 0,02 & $-0,07$ & $0,23^{*}$ & 1,00 \\
\hline
\end{tabular}

DMS: Drive for Muscularity Scale; RSS: Rosenberg Self-Esteem Scale; BDI: Beck Depression Inventory; EAT-26: Eating Attitudes Test-26; CES: Commitment Exercise Scale. * significativo para $p<0,05$.

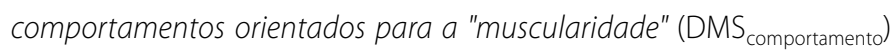
com os comportamentos de risco para transtornos alimentares e com o grau de comprometimento psicológico ao exercício físico.

Seguiu-se com a análise de regressão linear múltipla Forward a partir das associações demonstradas na Tabela 2. Para tanto, foram realizadas três regressões contando em cada uma com uma variável critério: busca pela "muscularidade" (DMS total $_{1}$, insatisfação com a "muscularidade" (DMS insatisfação) e comportamentos orientados para a "muscularidade" (DMS comportamento). Para essas análises, a ordem de inserção das variáveis no modelo de regressão respeitou a magnitude do coeficiente de correlação de Pearson do maior para o menor valor. Esses resultados estão demonstrados na Tabela 3. Destaca-se que independente das associações verificadas entre as variáveis (Tabela 2), em todos os três modelos de regressão a variável preditora com maior influência foi o grau de comprometimento psicológico ao exercício físico.

Tabela 3. Análises de regressão linear múltipla forward utilizando os escores da $\mathrm{DMS}_{\text {total }}, \mathrm{DMS}_{\text {insatisfacāo }}$ e $\mathrm{DMS}_{\text {comportamento }}$ como variáveis critério.

\begin{tabular}{c|c|c|c|c|c}
\hline & bloco & $\mathbf{R}^{\mathbf{2}}$ & $\mathbf{R}^{2}$ ajustado & $\mathbf{F}$ & $\mathbf{p}$-valor \\
\hline DMS $_{\text {total }}$ & & & & & \\
\hline CES & 1 & 0,387 & 0,384 & 153,794 & $0,0001^{*}$ \\
\hline RSS & 2 & 0,401 & 0,396 & 81,306 & $0,0001^{*}$ \\
\hline DMS $_{\text {insatisfaçäo }}$ & & & & & \\
\hline CES & 1 & 0,127 & 0,123 & 35,424 & $0,0001^{*}$ \\
\hline RSS & 2 & 0,146 & 0,139 & 20,702 & $0,0001^{*}$ \\
\hline BDI & 3 & 0,167 & 0,157 & 16,225 & $0,0001^{*}$ \\
\hline DMS $_{\text {comportamento }}$ & & & & & \\
\hline CES & 1 & 0,512 & 0,510 & 256,318 & $0,0001^{*}$ \\
\hline
\end{tabular}

CES: Commitment Exercise Scale; RSS: Rosenberg Self-Esteem Scale; BDI: Beck Depression Inventory. ${ }^{*}$ significativo para $p<0,05$.

\section{DISCUSSÃO}

São frequentes os estudos que avaliam a insatisfação corporal e a prevalência de transtornos alimentares em diversas populações ${ }^{8-15}$. Entretanto, esses estudos têm avaliado a busca pela magreza, de forma que existe uma carência sobre os estudos com a população masculina e sobre o construto busca pela "muscularidade". Esta pesquisa teve como objetivo avaliar atitudes e comportamentos que refletem o grau de preocupação dos indivíduos com sua quantidade de músculos e associá-la a demais variáveis, de maneira a contribuir para essa lacuna do conhecimento.

Segundo Olivardia et al. ${ }^{3}$, indivíduos do sexo masculino apresentam elevada preocupação com a "muscularidade" e interesse pelo desenvolvimento de regiões como ombro, peitorais e braços. Jovens universitários acreditam que seus corpos são menores do que realmente são, crença essa que está associada a sintomas depressivos, baixa autoestima, aos comportamentos de risco para transtornos alimentares, além da adoção de comportamentos orientados para o ganho muscular ${ }^{3}$.

Também na presente pesquisa, a busca pela "muscularidade" esteve associada à baixa autoestima, aos comportamentos de risco para transtornos alimentares e ao grau de comprometimento psicológico ao exercício. Já a insatisfação com a "muscularidade" demonstrou associação com a baixa autoestima, os sintomas depressivos e com o grau de comprometimento psicológico ao exercício físico.

A partir dos resultados da presente pesquisa, não foi verificada associação da insatisfação com a "muscularidade" e os comportamentos de risco para transtornos alimentares, ao contrário de Olivardia et al. ${ }^{3}$. É possível que esse resultado esteja relacionado aos diferentes instrumentos de medida utilizados. Além disso, sintomas depressivos parecem acometer apenas indivíduos com elevada insatisfação com a "muscularidade"3,4. Embora o instrumento de medida utilizado (DMS) não permita a classificação quanto a esse patamar, é possível observar que o escore médio obtido $(24,24 \pm 9,36)$ para a variável insatisfação $\left(\mathrm{DMS}_{\text {insatisfação }}\right)$ na população investigada foi baixo em relação à variação possível para essa subescala (7 a 42 pontos). 
Segundo alguns autores ${ }^{3,4,6,7,9}$, a busca pela "muscularidade" está associada a prática excessiva de exercício físico, ao uso de suplementos alimentares e esteroides anabólicos. E ainda, indivíduos insatisfeitos com a sua imagem corporal recorrem frequentemente a comportamentos considerados de risco para o desenvolvimento de transtornos alimentares como a restrição alimentar, uso de laxantes e diuréticos e autoindução à vômitos ${ }^{4,6}$.

Embora esses comportamentos pareçam contrários ao ganho de massa muscular é preciso destacar a importância de um baixo percentual de gordura. Ou seja, para que o músculo se destaque é preciso que o indivíduo apresente baixa gordura corporal, de forma que exista uma definição muscular ${ }^{5}$. Assim, é possível que em alguns períodos da vida, sujeitos insatisfeitos adotem comportamentos deletérios à saúde.

Quanto aos comportamentos orientados para o ganho muscular foi verificada associação dessa variável com a baixa autoestima, os sintomas depressivos e com o grau de comprometimento psicológico ao exercício físico. A busca incessante pela "muscularidade" leva os indivíduos a um elevado grau de dependência ao exercício físico e adoção de medidas extremas para o ganho de peso ${ }^{34,6-8}$, , como o uso de esteroides anabólicos ${ }^{8}$ e suplementos alimentares ${ }^{9}$.

Ocorre que o ideal de corpo hipermuscular determinado socialmente não é de fácil aquisição. Por essa razão, apenas alguns indivíduos conseguem atingir esse corpo, enquanto os demais desenvolvem insatisfação com o corpo 5 . Schmitz e Campagnolo' por exemplo, verificaram que 17,6\% dos praticantes de musculação apresentam risco para o desenvolvimento de Dismorfia Muscular, patologia caracterizada como uma forma de transtorno dismórfico corporal em que existe preocupação com a aparência corporal por inteiro, relacionado à força e quantidade de massa muscular?

A busca pela "muscularidade" é um construto complexo e multifacetado, que inclui ao menos a insatisfação com a quantidade de músculos e os comportamentos orientados para o ganho muscular. Associado ao construto podemos observar diversas variáveis como a autoestima, sintomas depressivos e comportamentos de risco para transtornos alimentares $3,4,6-9$, assim como confirmam os dados a presente pesquisa.

Entretanto, destaque se faz ao papel do grau de comprometimento psicológico ao exercício frente ao construto. Independente da associação dos construtos avaliados com as demais variáveis, foi o grau de comprometimento psicológico ao exercício que demonstrou maior influência sobre a busca pela "muscularidade", explicando 38,4\% da variância total da DMS total 12,3\% da DMS insatisfação $_{\text {e }} 51,0 \%$ da DMS comportamento.

Em conjunto esses dados alertam para os riscos à saúde da busca incessante por massa muscular, representada pela idealização social do corpo hipermusculoso. Indivíduos com elevada busca pela "muscularidade" estão sujeitos ao desenvolvimento de baixa autoestima, aparecimento de sintomas depressivos, adoção de comportamentos de risco para transtornos alimentares, e em especial, ao desenvolvimento de um elevado grau de comprometimento ao exercício físico.

A despeito das contribuições da presente pesquisa algumas limitações devem ser apontadas. Primeiro, foram utilizados instrumentos de autorrelado, que podem não ser a melhor estratégia para avaliação de algumas questões. Por exemplo, alguns indivíduos podem não responder fielmente a alguns itens do questionário (DMS), como por exemplo, "Penso em tomar esteroides anabolizantes"? No entanto, cabe ressaltar que o instrumento foi validado para a amostra de jovens adultos brasileiros e obteve adequadas propriedades psicométricas ${ }^{17}$. Em segundo lugar, pode ser apontado o corte transversal do estudo, o que impossibilita o estabelecimento de relação de causa e efeito entre as variáveis, de forma que se recomenda a realização de estudos longitudinais. E por fim, a seleção amostral não probabilística.

\section{CONCLUSÃO}

Pode-se concluir que a busca pela "muscularidade" está associada à baixa autoestima, aos comportamentos de risco para transtornos alimentares e ao grau de comprometimento psicológico ao exercício físico. Em especial essa última variável foi a que demonstrou maior influência sobre o construto drive for muscularity. Profissionais de saúde que lidam que essa população devem estar atentos à excessiva busca pelo ideal de corpo hipermusculoso e aos comportamentos deletérios à saúde advindos dessa busca.

Todos os autores declararam não haver qualquer potencial conflito de interesses referente a este artigo.

CONTRIBUIÇÕES DOS AUTORES: PHBC (0000-0002-4918-5080)* e MECF (0000-0002-3294-7560)* foram responsáveis pela idealização da pesquisa, participando de todas as etapas do trabalho. CMN (0000-0002-5240-6710)*, FCO (0000-0002-2773-9003)*, JFFM (0000-0001-8396-6449)* e VGVL (0000-0001-9342-2090)*participaram da coleta e análise dos dados, além de revisar o manuscrito e contribuiram com a escrita do mesmo. *Número ORCID (Open Researcher and Contributor ID).

\section{REREFÊNCIAS}

1. Swami V, Frederick DA, Aavik T, Alcalay L, Allik J, Anderson, D, et al. The attractive female body weight and female body dissatisfaction in 26 countries across 10 world regions: results of the international body project I. Pers Soc Psychol Bull. 2010;36(3):309-25.

2. Rodin J, Silberstein L, Striegel-Moore R. Women and weight: A normative discontent. In: Sonderegger TB, editor. Psychology and gender. Lincoln: University of Nebraska Press; 1985. p. 275-307.

3. Olivardia R, Pope Jr., HG, Borowiecki, JJ, Cohane, GH. Biceps and body image: The relationship between muscularity and self-esteem, depression, and eating disorder symptoms. Psychol Men Masculinity. 2004;5(2):112-20.

4. Cafri G, Thompson JK, Ricciardelli L, McCabe M, Smolak L, Yesalis C. Pursuit of the muscular ideal: Physical and psychological consequences and putative risk factors. Clin Psychol Rev. 2005;25(2):215-39.

5. Carvalho PHB, Ferreira MEC. Imagem corporal em homens: Instrumentos avaliativos. Psic Teor Pesq. 2014;30(3):277-85

6. Edwards $C$, Tod D, Molnar G. A systematic review of the drive for muscularity research area. Int Rev Sport Exerc Psychol. 2014;7(1):18-41.

7. McCreary DR, Sasse DK. An exploration of the drive for muscularity in adolescent boys and girls. J Am College Health. 2000;48(6):297-304.

8. Iriart JAB, Chaves JC, Orleans RG. Culto ao corpo e uso de anabolizantes entre praticantes de musculação: Cad Saúde Pública. 2009;25(4):773-82.

9. Schmitz JF, Campagnolo PDB. Características de dismorfia muscular em praticantes de musculação: Associação com o consumo alimentar. Braz J Sports Nutr. 2013;2(2):1-8.

10. Pereira Junior M, Junior WC, Silveira FV. Percepção e distorção da auto imagem corporal em praticantes de exercício físico: a importância do exercício físico na imagem corporal. Rev Bras Nutr Esport. 2013;7(42):345-52.

11. Oliveira FP, Bosi MLG, Vigário PS, Viera RS. Comportamento alimentar e imagem corporal em atletas. Rev Bras Med Esporte. 2003;9(6):348-56.

12. Hass AN, Garcia ACD, Bertoletti J. Imagem corporal e bailarinas profissionais. Rev Bras Med Esporte. 2010;16(3):182-85.

13. Guimarães AD, Machado SP, França AKTC, Calado IL. Transtornos alimentares e insatisfação com a imagem corporal em bailarinos. Rev Bras Med Esporte. 2014;20(4):267-71.
14. Fortes, LS, Neves CM, Filgueiras JF, Alameida SS, Ferreira MEC. Insatisfação corporal, comprometimento psicológico ao exercício e comportamento alimentar em jovens atletas de esportes estéticos. Rev Bras Cineantrop Desemp Hum. 2013;15(6): 695-704.

15. Neves CM, Fortes LS, Filgueiras JF, Ferreira MEC. Comportamentos alimentares em ginastas de elite: Associação com o perfeccionismo e o estado de humor. Rev Educ Física. 2013;24(3):359-69.

16. McCreary DR, Sasse DK, Saucier DM, Dorsch KD. Measuring the drive for muscularity: Factorial validity of the Drive for Muscularity Scale in men and women. Psychol Men Masculinity. 2004;5(1):49-58.

17. Campana ANN, Tavares MCG, Swami V, Silva D. An examination of the psychometric properties of brazilian portuguese translations of the Drive for Muscularity Scale, the Swansea Muscularity Attitudes Questionnaire, and the Masculine Body Ideal Distress Scale. Psychol Men Masculinity. 2013;14(4):376-88

18. Streiner DL. Starting at the beginning: an introduction to coefficient alpha and internal consistency. J Pers Assess. 2003;80(1):99-103.

19. Rosenberg M. Society and the adolescent self-image. Princeton, NJ: Princeton University Press; 1965

20. Hutz CS. Adaptação brasileira da escala de autoestima de Rosenberg [monografia]. Porto Alegre: Universidade Federal do Rio Grande do Sul; 2000.

21. Beck AT, Ward CH, Mendelson M, Mock J, Erbaugh J. An inventory for measuring depression. Arch Gen Psychiatry. 1961;4(6):561-71.

22. Gorestein C, Andrade L. Inventário de depressão de Beck: propriedades psicométricas da versão em português. Rev Psiquiatr Clin. 1998;25(5):245-50.

23. Garner DM, Olmsted MP, Bohr Y, Garfinkel PE. The eating attitudes test: psychometric features and clinical correlates. Psychol Med. 1982;12(4):871-78.

24. Nunes MA, Camey S, Olinto MTA, Mari JJ. The validity and 4-year test-retest reliability of the Brazilian version of the Eating Attitudes Test-26. Braz J Med Biol Res. 2005;38(11):1655-62.

25. Teixeira PC, Hearst N, Matsudo SMM, Cordás TA, Conti MA. Adaptação transcultural: tradução e validação de conteúdo da versão brasileira do Commitment Exercise Scale. Rev Psiquiatr Clin. 2011:38(1):24-8.

26. World Health Organization. WHO Expert Committee on Physical Status: the use and interpretation of anthropometry physical status. Geneva: World Health Organization; 1995 (WHO Technical Report Series, v. 854). 